\title{
Holographic and trace strength models of rehearsal effects in the item recognition task
}

\author{
PATRICK CAVANAGH \\ Universite de Montreal, Montreal, Quebec, Canada
}

\begin{abstract}
Two models are described that are capable of generating latency predictions in the item recognition task based on the sequence and timing of rehearsals. It is shown that equal positive and negative slopes are possible when the average number of rehearsals per item increases with list length but that when no rehearsal occurs the positive slope is predicted to be substantially steeper than the negative. Serial position effects are also analyzed for the various rehearsal strategies seen when subjects are asked to rehearse out loud.
\end{abstract}

In a 1966 paper, Sternberg proposed a serial exhaustive search model to account for the results he had found in his item recognition task: a linear relation between reaction time and memorized list length for both positive and negative responses, equal slopes of this linear relation for both response types, the absence of serial position effects. Although his results have been frequently replicated under a variety of conditions, contradictory findings have also been reported: significant serial position effects (Corballis, 1967), repetition effects (Baddeley \& Ecob, 1973), and positive slopes significantly steeper than negative (Corballis, Kirby, \& Miller, 1972). Such results have lead these authors and others (Nickerson, 1972; Clifton, Gutschera, Brewer, \& Cruse, Note 1) to propose trace strength models of memory search which appear to explain qualitatively many phenomena in the item recognition task.

In this paper, I would like to report the results of a quantitative analysis of two models capable of generating latency predictions in the item recognition task: a holographic model (Cavanagh, 1972a) and an extended trace strength model (Corballis, et al., 1972; Gescheider, Wright, Weber, Kirchner, \& Milligan, 1969; Nickerson,. 1972; Wickelgren, 1970a). Specifically, the investigation centers on the possibility that differences in rehearsal strategy are the source of the variations found in item recognition data.

The first hypothesis is that equal amounts of learning time for every item at all list lengths will lead to significantly steeper positive than negative slopes. For example, a large slope difference (positive $34 \mathrm{msec} /$ item, negative 24) was found by Wescourt and Atkinson (1973) when subjects were asked to rehearse list items aloud only once. Now, when no specific rehearsal instructions are given, it is not

This paper was presented in part at the meeting of the Midwestern Psychological Association, Chicago, May 10, 1973. The support of the National Research Council of Canada and the assistance of Susan Peters are gratefully acknowledged. possible to ascertain the strategies used by subjects-except, possibly, when list items are presented at such a rapid rate that rehearsal is either not possible or not necessary. In the absence of rehearsal. it can reasonably be assumed that all items receive equal amounts of learning time. Thus, steeper positive than negative slopes should also be seen in studies involving rapid presentation rates and short delays between the last item and the test. In fact, the three studies that do use rapid presentation (Aubé \& Murdock, 1974, .25 sec/item, .3-sec delay; Corballis, et al., 1972. .3 sec/item, .6-sec delay; Forrin \& Morin, 1969, .333 sec/item, .5-sec delay) all report a positive slope significantly greater than the negative.

With slightly slower presentation rates (e.g., $.5 \mathrm{sec} /$ item, Burrows \& Okada, 1971), positive and negative slopes return to approximate equality. The importance of the assumed introduction of rehearsal at slower rates when uncontrolled by instruction is that the sequential presentation of list items used in most studies biases subjects toward more rehearsal per item at longer list lengths. For example, in a list of length $n$, sequentially presented, the first item can be rehearsed $n$ times, the second $n-1$, the third $n-2$. and so on. The average number of opportunities to rehearse an item in a list of length $n$ is then $(n+1) / 2$. Thus, the equality of positive and negative slopes reported by Sternberg (1966) may well result from this possible increase in average learning time per item with list length.

Two hypotheses of the interaction between rehearsal strategy and serial position effects will also be analyzed. The first is related to decay ${ }^{1}$ of memory information and assumes that the lesser amount of decay of the most recent items (whether presentations or rehearsals) will result in faster reaction times for these items. With a rapid presentation rate and short delay, the temporal order of items prior to the test (assuming no rehearsal occurs) will be equivalent to their presentation order and thus be invariant over subjects and lists. Averaged data should therefore show reliable recency effects. Longer delays will 
permit substantial list rehearsal prior to the test. With fluctuations in the speed of rehearsal between trials and between subjects, the last list position rehearsed may vary in an essentially random fashion. Averaged data at longer delays might, therefore. show no recency effects. Second, it has been found in recall tasks that primacy results from additional rehearsals accorded the early items in a list (Rundus \&Atkinson, 1970). Corballis et al. (1972) also suggest that primacy in item recognition data might be due to extra rehearsals of initial list items. It is possible that this primacy, if it exists, might combine with recency effects under certain circumstances to produce a flat serial position curve. In any event, the elimination of recency seen at slow presentation rates and long delay must be directly related to rehearsal as instructions not to rehearse (Kirsner \& Craik, 1971, who used only list length eight) reintroduce large recency effects.

To investigate these rehearsal hypotheses [the analysis of the repetition effects shown by Baddeley and Ecob, 1973, has been given elsewhere (Cavanagh, Note 2)], two models were constructed to incorporate the sequence and timing of rehearsal into their reaction time predictions. Typical rehearsal protocols were determined by instructing subjects to rehearse out loud while performing the task. A brief description of the two models follows; both are described in more detail in other papers.

\section{HOLOGRAPHIC MODEL}

The basic premise of the holographic model is that symbolic information is presented by spatial patterns of neural activity. which may be termed wavefronts. If two wavefronts project to the same group of neurons. and these neurons are modified by the input they receive, then the resulting modifications of the neurons store the interference pattern of the two wavefronts. This stored interference pattern is the neural hologram and provides an associative memory- referencing the memory with either of the original wavefronts will reconstruct or retrieve the other. In addition, it is possible to superimpose many interference patterns on the same group of neurons while still allowing retrieval of individual associations. Other considerations essential to the holographic hypothesis are the neural code assumed, the linearity of transmission, and the neural transforms involved. These have been covered in a number of papers (Barrett, 1970; Cavanagh, 1972a; Swigert, 1971; van Heerden, 1963; Westlake, 1970).

Since the item recognition task involves short-term memory (STM), the modification required in the neurons storing the hologram need only be temporary. The most logical choice for the neural moditication is. therefore, simply the habituation or fatigue of the individual cells in response to the input each receives. Habituation is a decrease in the transmission efficiency of the cell, and the variation in habituation across the group of storage cells is analogous to the variation in opacity of film in an optical hologram. Since habituation follows an exponential function, we can derive mathematical expressions for the storage processes and for the strength of retrieved signals in terms of mean firing rates.

In the item recognition task, each element in the list to be memorized is stored as an association between its sensory representation and it internal representation in the following manner (Cavanagh, 1972a). Upon presentation of a list item. its sensory representation (wavefront) is simultaneously transmitted to both a long-term and a short-term store, as shown in Figure 1. At the long-term store, an internal code is generated for the item. This more abstract encoding of the item then propagates to the short-term memory where the sensory representation is concurrently incident. In this fashion-although there are a number of other possibilities-an interference pattern or association is stored for each list item. When a test stimulus is presented. it will reconstruct its internal representation (wavefront) only if the appropriate association has been stored, that is, only if it was a member of the memorized list. The detection of a reconstruction can therefore be used to initiate a positive response. A detection of the sensory representation after transmission through the storage cells, coupled with no detection of a reconstructed wavefront can initiate a negative response. Positive and negative responses are thus achieved by two separate but mutually inhibiting detectors.
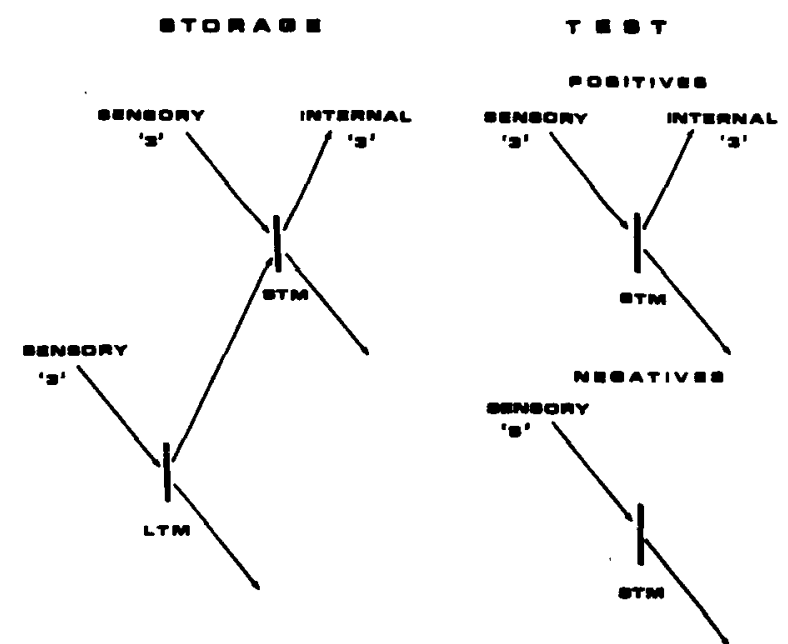

Figure 1. Storage and reconstruction in a holographic associative memory. Storage involves retrieval of an internal code from LTM followed by concurrent reception of sensory and internal codes at STM, effecting the storage of an interference pattern. To test memory for the presence or absence of a particular item, for example, a " 3 " or a " 5 ," the item is presented and the reconstructed and direct transmission signals are monitored. Detection of a reconstructed warefront indicates that the "3" was stored; detection of only the directly transmitted wavefront indicates that the "5" was not stored. 
The variation in reaction time $(\mathrm{RT})$ in this model lies in the change in latency required to detect the signal wavefronts, and this detection latency is assumed to be related to the strengths of the wavefronts. Since the transmission coefficients of the storage cells are exponentially related to the number of associations stored (i.e., the habituation of the cells increases exponentially with total exposure time), the average strength of the test stimulus's signal after passing directly through the storage cells will be a negative exponential function of the number of items, $\mathrm{n}$. in the memorized list. The efficiency of transmission to the reconstructed wavefront is determined by the amplitude of the stored interference pattern (i.e., the variation in transmission level-habituation-across the storage cells) that is specific to the input. The effect of exposing the storage cells to additional interference patterns is to reduce the amplitude, or contrast, of those already stored in a manner analogous to the overexposure of a film. That is, since there is a limiting value of habituation (zero transmission), cells that are already heavily habituated cannot greatly increase their habituation level, whereas those that are little habituated can. Thus the cell-to-cell variations in habituation must decrease as the overall level of habituation increases with subsequent exposure to other patterns. As with the overall level, this decrease in contrast, and therefore of the average strength of the reconstructed wavefront, is a negative exponential function of $n$, the number of stored patterns (Cavanagh, 1972a).

Finally, to determine the detection function relating the signal strength and reaction time, we need only look at the results of Sternberg's (1967) study involving visual noise. He found that the effects of visual noise and list length on $\mathrm{RT}$ were additive. In the holographic model, however, signal strength at the respective detectors is given by the product of the input strength to the holographic memory and the coefficients of transmission to the direct or reconstructed wavefronts. Only the input signal strength is affected by noise variables, while only the transmission coefficients are affected by list length. To transform this multiplicative relation to an additive one, the detection function must therefore be logarithmic. ${ }^{2}$

The combination of exponential and logarithmic functions leads to a prediction of linear RT variation with list length. The model also predicts equal positive and negative slopes and an inverse relationship between slope and memory span (Cavanagh, 1972a, b). This previous work, however, did not take into account the decay of habituation that returns a cell to its normal operating level, nor how this might interact with various rehearsal strategies.

In generating predictions for rehearsal strategies, the sequence of rehearsals will be given by $r_{n v m}$, where $r_{n v m}$ equals one if, for list length $n$, the item from serial position $v$ in the list was the $m^{\text {th }}$ rehearsal (an item's first recitation upon presentation and its subsequent rehearsals will be assumed to be equivalent events), and $r_{n v m}$ equals zero otherwise. Now

$$
\underset{\mathbf{v}}{\sum} \sum_{\mathbf{m}} r_{\mathbf{n v m}}=r_{n}
$$

is the total number of item rehearsals in the rehearsal protocol for list length $n$. The sequence of interrehearsal time intervals for the list length $n$ protocol will be given by $t_{n m}$. For notational convenience in the following equations, the time preceding the first rehearsal will be considered infinite $\left(t_{n o}=\infty\right)$.

Since habituation is an exponential function, the time change of the transmission coefficient, $\tau$ (omitting subscripts for the moment), for a constant level, $\mathrm{s}_{\mathrm{v}}$, of input to the neuron is given by the starting value, $\tau_{z}$, multiplied by the negative exponential function of the input energy, the product of the input level, and the input duration, $t_{v}$,

$$
\tau=\tau_{\mathbf{z}} \cdot \mathrm{e}^{-\mathbf{s}_{\mathbf{v}} \mathrm{t}_{\mathbf{v}}} .
$$

This simple formulation covers a number of possible physiological bases of habituation-slow buildup of self-inhibition (Wickelgren, 1967), transmitter depletion (Thies, 1965), or blockage of $\alpha$ receptor sites (Axelrod, 1971). Most importantly, however, it demonstrates that the effect of new input on the previous level of transmission is multiplicative.

For simplicity, the habituation expressions will be developed for a single neuron and the variations of the transmission coefficient from cell to cell will be analyzed later when average values are computed. The effect of the $v^{\text {th }}$ input, $s_{v} t_{v}$, will be represented as

$$
\mathrm{s}_{\mathrm{v}} \mathrm{t}_{\mathrm{v}}=\alpha_{\mathrm{o}} \pm \alpha_{\mathrm{v}},
$$

where $\alpha_{0}$ is the average input energy over all the neurons in the storage area and $\alpha_{\mathrm{v}}$ is the spatially variable portion of the input energy. For optimum storage, the magnitude of $\alpha_{v}$ should be a constant across all neurons (i.e., vary only in sign) and should also equal that of $\alpha_{0}$ (Cavanagh, 1972a). Furthermore, assuming homogeneity of the stimulus items and their storage times, $\alpha_{0}$ and $\alpha_{v}$ are independent of $v$.

The effect of the $m^{\text {th }}$ rehearsal, then, is to further habituate the storage cells, reducing the transmission coefficient from the level reached at the end of the $m$ - Ith interrehearsal interval, $\tau(n, m-1)$. Letting the item from the $\mathrm{v}^{\text {th }}$ list position be the $\mathrm{m}^{\text {th }}$ rehearsal and letting $\tau^{\prime}(\mathrm{n}, \mathrm{m})$ represent the transmission coefficient immediately after the $m^{\text {th }}$ rehearsal, we have

$$
\tau^{\prime}(\mathrm{n}, \mathrm{m})=\tau(\mathrm{n}, \mathrm{m}-1) \cdot \mathrm{e}^{-\alpha_{\mathrm{o}} \pm \alpha_{\mathrm{v}}} .
$$


The introduction of dishabituation complicates the mathematical expressions; however, the duration of storage (probably on the order of $100 \mathrm{msec}$ ) will be assumed short enough so that the effects of dishabituation during storage can be ignored. Thus, storage will be viewed as an immediate event followed by an exponential return of cell transmission coefficients to their resting level (in this case, a level of 1 is assumed and $0 \leqslant \tau \leqslant 1)$. The dishabituation during the interrehearsal interval, $\mathrm{t}_{\mathrm{nm}}$, is therefore given by

$$
\tau(\mathrm{n}, \mathrm{m})=\tau^{\prime}(\mathrm{n}, \mathrm{m}) \cdot \mathrm{e}^{-\beta t_{n \mathrm{~m}}}+\left(1-\mathrm{e}^{-\beta t_{n m}}\right) .
$$

Combining Equations 1 and 2 recursively to obtain the final value of the transmission coefficient at the presentation of the test probe,

$$
\begin{aligned}
& \tau\left(n, r_{n}\right)=\left(1-e^{-\beta t_{n r_{n}}}\right)+\sum_{m=1}^{r_{n}}\left(1-e^{-\beta t_{n m}}\right) \\
& \cdot \mathrm{e}^{-\alpha_{0}\left(r_{n}-m\right)-\beta} \sum_{m=1}^{r_{n}} t_{n i}
\end{aligned}
$$

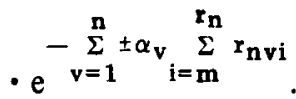

The negative reaction time depends, as described before, on the average transmission level of the storage neurons $-\bar{\tau}_{\mathrm{o}}(\mathrm{n})$ for list length $\mathrm{n}$. Only the final exponential term of Equation 3 is spatially varying. Since all input patterns are assumed to be independent and $+\alpha_{v}$ and $-\alpha_{v}$ occur with equal frequency in a pattern, the average value of this final term is

$\prod_{v=1}^{n}\left(\frac{e^{+\alpha v_{i=m} \sum_{n} r_{n v i}}+e^{-\alpha_{v} \sum_{i=m}^{r_{n}} r_{n v i}}}{2}\right)$

$$
=\prod_{\mathrm{v}=1}^{\mathrm{n}}\left[\cosh \left(\alpha_{\mathrm{v}} \sum_{\mathrm{i}=\mathrm{m}}^{\mathrm{r}_{\mathrm{n}}} \mathrm{r}_{\mathrm{nvi}}\right)\right] .
$$

Thus,

$$
\begin{aligned}
& \tau_{o}(n)=\left(1-e^{-\beta t_{n r_{n}}}\right)+\sum_{m=1}^{r_{n}}\left(1-e^{-\beta \cdot t_{n m}-1}\right) \\
& \mathrm{e}^{-\alpha_{0}\left(r_{n}-m\right)-\beta} \sum_{m=1}^{r_{n}} t_{n i} \\
& \prod_{v=1}^{n}\left[\cosh \left(\alpha_{v} \sum_{i=m}^{r_{n}} r_{n v i}\right)\right] .
\end{aligned}
$$

Upon presentation of a test stimulus whose neural strength (i.e., the average amplitude of the activity pattern coding the stimulus) is $\mathbf{A}_{\mathbf{o}}$, the strength of the signal pattern directly transmitted through the storage cells is

$$
\mathbf{s}_{\mathrm{o}}=\mathrm{A}_{\mathrm{o}} \cdot \bar{\tau}_{\mathrm{o}}(\mathrm{n}) \text {. }
$$

Letting $a_{1}$ represent the neural transmission and response execution times, and $\mathrm{k}$ the constant of proportionality relating the logarithm of neural strength to detection time, the negative reaction time is, then,

$$
\begin{aligned}
\operatorname{RT}^{-}(n) & =a_{1}-k \cdot \log \left[A_{o} \cdot \bar{\tau}_{o}(n)\right] \\
& =a_{1}-k \cdot \log \left(A_{o}\right)-k \cdot \log \left[\bar{\tau}_{o}(n)\right] .
\end{aligned}
$$

Since the intensity of the test stimulus and, therefore, $A_{0}$ is independent of list length, its effect can be included in an overall constant $\mathrm{a}^{-}$,

$$
\mathrm{RT}^{-}=\mathrm{a}^{-}-\mathrm{k} \cdot \log \left[\bar{\tau}_{\mathrm{o}}(\mathrm{n})\right] .
$$

The strength of the reconstructed signal on input of the vth item as a test stimulus is given by the average variation of transmission coefficient specific to the input. Again in Equation 3, only the final exponential term varies spatially. The variation of this term specific to the $v^{\text {th }}$ interference pattern is, averaging over variations due to the other $n-1$ patterns,

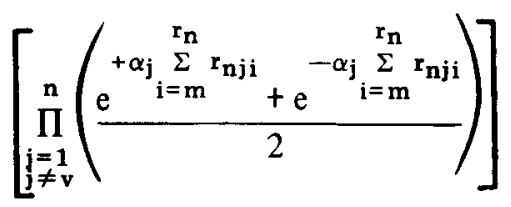

$$
\begin{array}{r}
\cdot\left(\frac{\left.e^{+\alpha_{v} \sum_{i=m}^{r_{n}} r_{n v i}-\alpha_{v} \sum_{i=m}^{r_{n}} r_{n v i}}\right)}{2}\right) \\
=\left\{\prod_{\substack{j=1 \\
j \neq v}}^{n}\left[\cosh \left(\alpha_{j} \sum_{i=m}^{r_{n}} r_{n j i}\right)\right]\right\} \cdot \sinh \left(\alpha_{v} \sum_{i=m}^{r_{n}} r_{n v i}\right) .
\end{array}
$$

Noting that the first term of Equation 3 does not vary spatially, the reconstruction transmission coefficient $\bar{\tau}_{v}(n)$ for the $v^{\text {th }}$ item is

$$
\begin{aligned}
\bar{\tau}_{v}(n) & =\sum_{m=1}^{r_{n}}\left(1-e^{-\beta t_{n m}-1}\right) \cdot e^{-\alpha_{o}\left(r_{n}-m\right)-\beta} \underset{m=1}{\sum_{m} t_{n i}} \\
\cdot & \left\{\prod_{\substack{j=1 \\
j \neq v}}^{n}\left[\cosh \left(\alpha_{j} \sum_{i=m}^{r_{n}} r_{n j i}\right)\right]\right\} \cdot \sinh \alpha_{v} \sum_{i=m}^{r_{n}} r_{n v i}
\end{aligned}
$$


Thus, the rehearsal of the $v^{\text {th }}$ item, although increasing the average habituation level, also increases the variations in habituation that encode it, therefore augmenting its stored representation.

The strength of the reconstructed signal upon input of $A_{v}$ is

$$
\mathbf{s}_{\mathbf{v}}=\mathbf{A}_{\mathbf{v}} \cdot \bar{\tau}_{\mathbf{v}}(\mathrm{n})
$$

and the positive reaction time is

$$
\begin{aligned}
\mathrm{RT}^{+}(\mathrm{n}) & =\mathrm{a}_{2}-\mathrm{k} \cdot \log \left[\mathrm{A}_{\mathrm{v}} \cdot \bar{\tau}_{\mathrm{v}}(\mathrm{n})\right], \mathrm{a}_{2} \text { a constant } \\
& =\mathrm{a}_{2}-\mathrm{k} \cdot \log \left(\mathrm{A}_{\mathrm{v}}\right)-\mathrm{k} \cdot \log \left[\bar{\tau}_{\mathrm{v}}(\mathrm{n})\right]
\end{aligned}
$$

Assuming that there are no encoding effects-i.e., that this is the first memory through which the neural activity pattern has passed that is influenced by the learning of the list items $-\mathbf{A}_{\mathbf{v}}$ will not be a function of list length, nor will it differ from $A_{o}$. Combining the two constants as $\mathrm{a}^{+}$gives

$$
\mathrm{RT}^{+}(\mathrm{n})=\mathrm{a}^{+}-\mathrm{k} \cdot \log \left[\bar{\tau}_{\mathrm{v}}(\mathrm{n})\right] .
$$

The constant of proportionality, $k$, has been set equal for both positive and negative reaction times. This is justified by the equality of the effect of stimulus degradation (which would affect $k \cdot \log A_{o}$ and $\mathrm{k} \cdot \log \mathrm{A}_{\mathrm{v}}$ but not $\left.\mathrm{k} \cdot \log \tau\right)$ on positive and negative responses as shown by Sternberg (1967).

Although these equations are fairly complex, there is essentially only one free parameter, $\alpha$, in the model. $\mathrm{a}^{+}$and $\mathrm{a}^{-}$, as additive constants, are of little interest here; $k$, the constant of proportionality, has been determined previously from simple RT tasks with variable stimulus intensity to be about $120 \mathrm{sec}$ (Cavanagh, 1972a); $\beta$, the time constant for decay or dishabituation, will be set at $.1 \mathrm{sec}^{-1}$, approximately the value found by Wickelgren $\left(1970 \mathrm{a} ; .13 \mathrm{sec}^{-1}\right)$ and Wickelgren and Berian $\left(1971 ; .08 \mathrm{sec}^{-1}\right)$ for the equivalent parameter in a number of recognition tasks. Only $\alpha$, the storage parameter, will be allowed to vary. Its value has also been determined previously from memory span measurements (Cavanagh, 1972a), but this earlier derivation treated the cumulative effect of rehearsals as a single storage and did not take into account dishabituation.

\section{EXTENDED TRACE STRENGTH MODEL}

The discrepancies between experimental results and the predictions that would be made by Sternberg's serial exhaustive search model (Sternberg, 1966) have led a number of people to propose various trace strength models of memory search (Baddeley \&Ecob, 1973; Corballis et al., 1972; Clifton, Gutschera, Brewer, \& Cruse, Note 1). Nickerson (1972) has put forth such a model in some detail. giving the following four axioms: (1) The time required to decide that an item is in the positive set is an inverse function of the trace strength for that item. (2) The time required for a negative decision is determined by the minimum strength that the relevant trace could be assumed to have if the probe had been a positive one; that is, it is slower than the slowest possible positive and so is classified essentially by default. (3) Trace strength from different rehearsals of the same item are cumulative. (4) Trace strengths decay exponentially with time. An investigation of this model by Ellis (Note 3), however, revealed several inadequacies. The most serious shortcoming is that the slope for negative responses is always greater than that for positives. In simulating the model for this paper. I found that the negative slope was consistently about twice as steep as the positive; other characteristics were poor linearity, wide variation in slopes in response to slight changes in rehearsal protocols, and, frequently, decreasing reaction time with increases in list length.

I therefore abandoned Nickerson's model and attempted to construct a memory search model from Wickelgren's (1970a, b, 1971) trace strength hypothesis in combination with proposals for latency predictions made by Corballis et al. (1972), Gescheider et al. (1969), and Norman and Wickelgren (1969). There are a number of major differences between this model and Nickerson's. First, an item's strength decays not only as a function of time, but also as a function of the number of subsequent items intervening between presentation and test (Wickelgren, 1970a). Second, in accord with signal detection theory, positive responses are generated for items with trace strengths exceeding a response criterion and negative responses for those with trace strengths below the criterion. The closer the item's trace strength is to the criterion, the slower the response (Norman \& Wickelgren, 1969). Third, the response criterion varies as a function of list length (Corballis et al.. 1972), being placed between the average trace strength for positive set items and the average trace strength for negative items. ${ }^{3}$ (The position of the criterion between the two averages is assumed a function of response biases.) If, on the other hand, the criterion is assumed not to vary with list length, it is easily shown that both reaction time and error rate for negative probes will decrease with list length. Fourth, Gescheider et al. (1969) have proposed that absolute trace strength, in addition to the criterion to trace strength difference, is an important factor in predicting latency in signal detection models. In justification, they cite the decrease in reaction time with increasing stimulus intensity in simple RT tasks. It is not clear to what extent absolute trace strength might influence $\mathrm{RT}$, but the inclusion of the proposal in this model is seen as a means of determining its importance.

This extended trace strength (ETS) model is thus 
described by the following axioms: (1) Positive RT is monotonically decreasing with the distance of the test item's trace strength above the criterion. (2) Negative $\mathrm{R} T$ is monotonically decreasing with the distance of the test item's trace strength below the criterion. (3) Both positive and negative RT decrease monotonically with the absolute trace strength of the test item. (4) The response criterion is determined independently on each trial. being placed at some position between the average trace strength of positive items and the average trace strength of negative items. both neasured at the time of the test item's presentation. (5) Trace strength decays exponentially with tine. (b) Trace strength is a decreasing exponential function of the number of intervening items. (7) Trace strengths are independent (Wickelgren. 1970b). (8) An item's trace strength is a monotonically increasing function of the number of repetitions of that item (Wickelgren, 1971). (9) Decay rates are invariant with the number of repetitions (Wickelgren. 1971).

Betore precise RT expressions are possible for the model, some assumptions are necessary:

(1) Negative logarithms will be used for the monotonically decreasing functions described in axions 1.2 , and 3 . Various negative power functions were also tried. but these could not generate the desired linear RT/list-length relations. In view of the exponential decay functions, the use of logarithms is reasonable (albeit ad hoc) in attempting to achieve linearity.

(2) Trace strengths from repetitions of the same item will be assumed to add linearly. This follows directly from axiom 7, which proposes the independence of trace strengths. This combination of linear addition of trace strengths and logarithmic relation between $\mathrm{RT}$ and strength is supported by the data of Baddeley and Ecob (1973) where RT can be show $n$ to be linearly related to the logarithm of the number of repetitions given an item in the positive set (Cavanagh, Note 2).

(3) Since the simulation of this model involves only single trials (with list length varying from one to six items, inclusively, throughout), the sequence of occurrences of a negative test item in preceding trials is not available. To overcome this. the expected trace strength of an item not in the current list is computed assuming an intinite number of trials preceding the present one. the equiprobability of list lengths one through six on each trial, and the random choice of list elements from the stimulus population on each trial. This expected residual trace strength is also added to all items in the positive set to reflect their occurrence in previous trials. One unknown factor in determining the effect of preceding trials is the iniportance of the test item: how much does it count as an intervening item. does its presentation augment the trace strength of the item, and is the effect different for a positive vs. a negative probe? These questions are beyond the scope of this simulation. For convenience, then, the presentation of an item as a test will be assumed not to influence the item's trace strength. ${ }^{4}$ The effect of its occurrence as an intervening event will be lumped in with the intertrial interval (ITI) duration to give a parameter. ' $t$. representing the overall decay produced by the test item and the ITI.

The strength, $s_{c}(v, n)$, of the $v^{\text {th }}$ item in a list of $n$ items, with the total number of rehearsals of all items equaling $r_{n}$, is, ignoring the trace strength due to occurrences in previous trials,

$$
S_{c}(v, n)=\sum_{m=1}^{r_{n}} r_{n v m} \cdot e^{-\alpha\left(r_{n}-m\right)-\beta} \stackrel{\substack{r_{n} \\ i=m}}{t_{n i}},
$$

where $r_{n v m}$ and $t_{n i}$ are as defined before, $\alpha$ is the item decay constant, and $\beta$ the time decay constant. Thus the mean trace strength, $\overline{\mathrm{s}}$, of an item at the end of a trial (including $t^{\prime}$, the effect of the probe and the intertrial interval) is, averaging over both serial position and list length (which varies from one to six),

$$
\bar{s}=\frac{1}{6} \sum_{n=1}^{6}\left[\sum_{v=1}^{n} s_{c}(v, n) \cdot p(v, n) \cdot e^{-\beta t^{\prime}}\right],
$$

where $p(v, n)$ is the probability of an item being in the $v^{\text {th }}$ position of a list of length $\mathrm{n}$. But

$$
\mathrm{p}(\mathrm{v}, \mathrm{n})=\frac{1}{\rho},
$$

where $\rho$ is the size of the stimulus population from which the list elements are randomly chosen. Thus,

$$
\bar{s}=\frac{e^{-\beta t^{\prime}}}{6 \rho} \cdot \sum_{n=1}^{6} \sum_{v=1}^{n} s_{c}(v, n) .
$$

Equation 10 gives the contribution to an item's trace strength of an average trial. If trials are strung out in an infinite sequence, the contribution from each trial decays as a function of the duration and number of intervening items of subsequent trials. The decay, $d(n)$, caused by an intervening trial of list length $n$, but not including the ITI-probe effect, is

$$
\mathrm{d}(\mathrm{n})=\mathrm{e}^{-\alpha \mathbf{r}_{\mathbf{n}}-\beta} \sum_{\mathbf{m}=1}^{\mathbf{r}_{\mathbf{n}}} \mathrm{t}_{\mathrm{nm}} .
$$

The average decay, independent of list length, and including the ITI-probe effect is, then, $\bar{d}$,

$$
\bar{d}=\frac{e^{-\beta t^{\prime}}}{6} \cdot \sum_{n=1}^{6} d(n) .
$$


At the time of the presentation of the test item for the current list, the average residual trace strength of the test item due to occurrences in all previous trials is, thus, $\mathrm{s}_{\mathbf{r}}(\mathrm{n})$,

$$
\begin{aligned}
s_{r}(n) & =\left(\overline{s d}+\overline{s d}^{2}+\overline{s d}^{3}+\cdots\right) \cdot d(n) \\
& =\frac{\overline{s d}}{1-d} \cdot d(n) .
\end{aligned}
$$

$\mathrm{s}_{\mathrm{r}}(\mathrm{n})$ is therefore the expected trace strength of an item that has not been presented in the current list. If an item has been presented in the current list (i.e., a positive item), its total trace strength, $s_{t}(v, n)$, is

$$
s_{t}(v, n)=s_{c}(v, n)+s_{r}(n) .
$$

The criterion, $c(n)$, is now placed at some position, $\gamma$, between the average trace strength for positive items and the average for negative items ( $\gamma$ can vary between 0 and 1 ),

$$
c(n)=\gamma \cdot\left[\frac{1}{n} \cdot \sum_{v=1}^{n} s_{t}(v, n)-s_{r}(n)\right]+s_{r}(n) .
$$

Positive reaction time for the vth item in a list of length $\mathrm{n}$ is now given by

$$
\mathrm{RT}_{\mathrm{v}}^{+}(\mathrm{n})=\mathrm{a}^{+}-\mathrm{k}_{1} \log \left[\mathrm{s}_{\mathrm{t}}(\mathrm{v}, \mathrm{n})\right]-\mathrm{k}_{2} \log \left[\mathrm{s}_{\mathrm{t}}(\mathrm{v}, \mathrm{n})-\mathrm{c}(\mathrm{n})\right],
$$

where $k_{1}$ and $k_{2}$ are constants of proportionality. Negative RT is

$$
\mathrm{RT}^{-}(n)=\mathrm{a}^{-}-\mathrm{k}_{1} \log \left[\mathrm{s}_{\mathrm{r}}(n)\right]-\mathrm{k}_{2} \log \left[\mathrm{c}(n)-\mathrm{s}_{\mathrm{r}}(\mathrm{n})\right] .
$$

In these equations, $\alpha$ and $\beta$, the item and time decay parameters, will be set at .15 items $^{-1}$ and $.1 \mathrm{sec}^{-1}$, respectively. These are approximately the values obtained by Wickelgren (1970a; $\alpha=.14, \beta=.13)$ and Wickelgren and Berian $(1971 ; \beta=.08)$. The free parameters are then $\rho$, the stimulus population size, $t^{\prime}$ the intertrial interval and test item presentation effect, $\gamma$ the criterion position, $k_{1}$ and $k_{2}$ the relative influence on RT of absolute trace strength vs. that of the trace strength to criterion difference, and $\mathrm{a}^{+}$and $\mathrm{a}^{-}$which again are not of interest here.

\section{COMPARISON OF THE HOLOGRAPHIC AND ETC MODELS}

It is reasonable to ask whether or not the two models described here are, in fact, different. The holographic model represents a short-term, asso- ciative memory system including storage and retrieval mechanisms, and a simple neural logic for determining whether a test stimulus has or has not been stored in the memory. The ETC model, on the other hand, represents a decision process that assumes that the "strength" of a memory trace can be directly accessed in order to determine whether a stimulus is old or new. Although the dynamics of the strength variable are explicit, no assumptions are made concerning the type of memory storage or the retrieval mechanisms. As described, then, the ETC model could just as well be representing a holographic memory as any other type. The dynamics of the unspecified ETC memory do, however, differ in one real sense from those derived for the holographic memory.

In the holographic model, the transmission coefficient for a particular item is influenced by all items preceding and following it; while in the extended trace strength model, an item's trace strength is influenced only by the items following it. This greater independence between memory representations should be most evidence in the predicted serial position effects where, for the same rehearsal protocol, the holographic model will necessarily predict smaller item-to-item $R T$ variation than the ETS model.

On first glance, there also appears to be a significant difference between the decision processes of the two models. specifically in their treatment of negative reaction times. Negative RT is a function of the average transmission coefficient of the positive set items in the holographic model, whereas it is a function of the residual trace strength of the negative test item in the ETS model. This difference, however, reflects only the simplifying assumption made for the holographic model that the residual associations for negative items are negligible. If these residual associations were taken into account, or, conversely, if the residual strengths of negative items in the ETC model were also assumed to be negligible, the decision processes of the two models would become functionally quite similar. In any case, the data of the varied set procedure that will be investigated are insensitive to the effects of the negative items; consequently, the differences, real and artiticial, between the two models' decision processes will have no significant effects here.

In spite of their differences, both models have at their core very similar bases-compare Equations 4 and 6 to Equation 9-that is, the negative exponential functions of the number of items and presentationtest delay. Before investigating the effects of the similarities and differences, I will outline the general pattern of results that has been reported for the item recognition task and describe a preliminary study of rehearsal protocols. 
Table 1

Summary of Results in the Item Recognition Experiment

\begin{tabular}{|c|c|c|c|c|c|c|c|c|}
\hline \multirow[b]{2}{*}{ Source } & \multirow[b]{2}{*}{$\begin{array}{l}\text { Presentation } \\
\text { Mode }\end{array}$} & \multirow[b]{2}{*}{$\begin{array}{l}\text { Presenta- } \\
\text { tion Rate } \\
\text { (sec/item) }\end{array}$} & \multirow[b]{2}{*}{$\begin{array}{c}\text { Delay } \\
\text { Interval } \\
\text { (sec) }\end{array}$} & \multirow[b]{2}{*}{$\begin{array}{l}\text { List Length } \\
\text { and Material }\end{array}$} & \multicolumn{3}{|c|}{ Slopes } & \multirow[b]{2}{*}{ Serial Position Effects } \\
\hline & & & & & $\begin{array}{c}\text { Pos } \\
\text { (ms/ } \\
\text { item) }\end{array}$ & $\begin{array}{c}\text { Neg } \\
\text { (ms/ } \\
\text { item) }\end{array}$ & $\begin{array}{l}\text { Diffe- } \\
\text { rence }\end{array}$ & \\
\hline Ellis \& Chase, 1971 & Simultaneous & $\dagger$ & 1.000 & $1-4$ Letters & 49 & 38 & n.s. & $\begin{array}{l}\text { Gradual primacy at } \\
\text { List Length } 4\end{array}$ \\
\hline Cavanagh \& Chase, 1971 & Simultaneous & $\dagger$ & $\begin{array}{l}1.000 \\
4.500\end{array}$ & 1-6 Letters & 32 & 27 & n.s. & $\begin{array}{l}\text { Flat } \\
\text { Flat }\end{array}$ \\
\hline Clifton \& Birenbaum, 1970 & Sequential & 1.500 & $\begin{array}{r}2.500 \\
.500\end{array}$ & 1-7 Digits & 28 & 35 & n.s. & $\begin{array}{l}\text { Flat } \\
\text { Recency }\end{array}$ \\
\hline Burrows \& Okada, 1971 & Sequential & $\begin{array}{r}1.200 \\
.500\end{array}$ & $\begin{array}{r}2.400 \\
.500\end{array}$ & 1-4 Digits & $\begin{array}{l}26 \\
33\end{array}$ & $\begin{array}{l}35 \\
28\end{array}$ & $\begin{array}{l}\text { n.s. } \\
\text { n.s. }\end{array}$ & $\begin{array}{l}\text { Recency } \\
\text { Recency and primacy }\end{array}$ \\
\hline $\begin{array}{l}\text { Sternberg, } 1966 \\
\text { Forrin \& Morin, } 1969\end{array}$ & $\begin{array}{l}\text { Sequential } \\
\text { Sequential }\end{array}$ & $\begin{array}{r}1.200 \\
.333\end{array}$ & $\begin{array}{r}2.000 \\
.500\end{array}$ & $\begin{array}{l}\text { 1-6 Digits } \\
\text { 1-3 Letters }\end{array}$ & $\begin{array}{l}43 \\
54\end{array}$ & $\begin{array}{l}33 \\
26\end{array}$ & $\begin{array}{c}\text { n.s. } \\
\mathrm{p}<.001\end{array}$ & $\begin{array}{l}\text { Flat } \\
\text { Steep recency }\end{array}$ \\
\hline Corballis et al., 1972 & Sequential & .300 & .600 & $1-4,6$ Letiers & 41 & 27 & $\mathrm{p}<.01$ & $\begin{array}{l}\text { Strong recency } \\
\text { and primacy }\end{array}$ \\
\hline Aubé \& Murdock, 1974 & Sequential & .250 & .300 & 1-8 Digits & $23^{*}$ & $16^{* *}$ & $\mathrm{p}<.05^{* *}$ & $\begin{array}{l}\text { Strong recency } \\
\text { and primacy }\end{array}$ \\
\hline
\end{tabular}

*Aubé and Murdock (1974) analyzed only List Lengths 3 to 8 and reported positive and negative slopes of 19 and 8 msec/item, respectively. The slope values given here are based on data from List Lengths 1 through 8 inclusive.

**List Lengths 3 to 8 only. Significance level would increase for a slope comparison of Lengths 1 to 8 inclusive.

†Subject viewed list until ready.

\section{SURVEY OF EXPERIMENTAL RESULTS}

Experimental manipulations such as sequential vs. simultaneous presentation of list items, rate of list presentation, and memorization-test delay all may indirectly influence rehearsal. To determine if they might, in turn, affect RT, studies that have reported positive and negative slopes along with serial position effects are listed in Table 1 . The first point to notice is that there is no clear-cut difference between results for simultaneous and sequential presentation. The second is that all of these studies but one have a positive slope greater than the negative. This also holds true for most other studies that have not been included here. Frequently, this difference is not significant and the slopes are consequently reported as equal. The overall impression when all these studies are combined is. however, that positive slope is greater than the negative. A more important point here is that only at fast presentation rates, where it is possible that there is no rehearsal, does the positive-negative slope difference become significant.

Finally, serial position curves do show a relation to memorization-test delay. That is, a recency effect can be reliably produced with delays of less than $1 \mathrm{sec}-$ although a delay of more than $1 \mathrm{sec}$ does not guarantee the absence of recency. Primacy effects can be obtained sometimes, mostly at long list lengths and rapid presentation rates. One other important effect that appears reproducible is that the RT for the last serial position in a list of three items or more remains constant or even decreases as list length increases (Burrou's \& Okada. 1971; Corballis et al., 1972). Figure 2 shows the serial position curves from Corballis et al. (1972).

\section{REHEARSAL PATTERNS}

To investigate the type of rehearsal strategies employed in the item recognition task and their interaction with presentation mode and rate, an informal study of three subjects was run using overt rehearsal but not measuring reaction times. It was thought that the instructions might bias subjects to produce more rehearsal than would normally occur simply because of the attention it was receiving. For this reason, a very fast presentation condition, where the distortion of normal rehearsal would be greatest. was not included. Although limited in scope, this study produced some useful results.

First of all, there was a large variation in strategy between subjects: two relaxed subjects rehearsed a

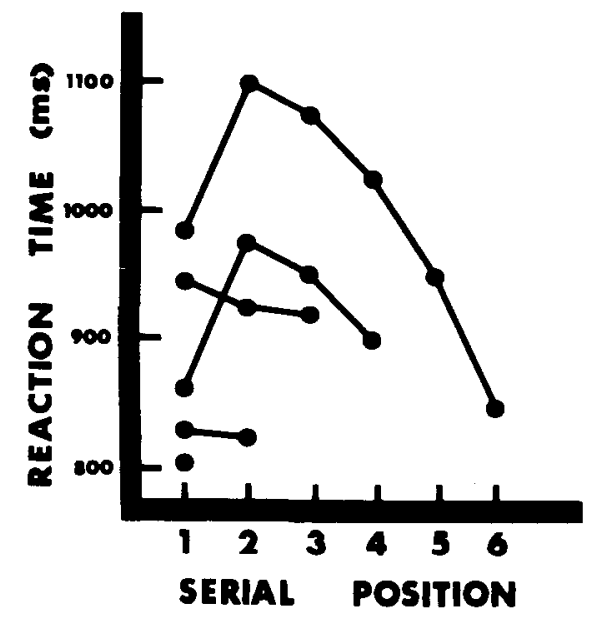

Figure 2. Serial position effects reported by Corbalis et al. (1972). Presentation rate was $.3 \mathrm{sec} /$ item and the delay interval was .6 sec. 
moderate amount and only, they said, when they felt it necessary for retention of the list; the third subject rehearsed pretty much all the time, though this might have been a function of the overt rehearsal instructions.

The two presentation modes did not produce the expected differences in rehearsal. In simultaneous presentation, the whole list is immediately available for rehearsal, whereas in sequential presentation, the list becomes available one item at a time, biasing the subject toward partial rehearsals and toward more rehearsal at longer list lengths. The actual difference found was slight, however, as subjects tended to group items in the same manner in both modes. For example, with simultaneous presentations, subjects would often rehearse the first three items once or twice and then the full list once or twice, while in sequential presentation, they frequently waited until the first three items had been exposed, then rehearsed them as a group. adding new items as they appeared.

The results from these subjects offered some general guidelines for rehearsal protocols. When it occurs, rehearsal within interitem intervals and delay periods is typically one or more repetitions of the whole list (list-to-date); only rarely does the next item or the test item disrupt an ongoing list rehearsal. Items within rehearsals of longer lists are sometimes grouped in twos or threes with a slight pause between groups. Later rehearsals appear more rapid than the first. At faster presentation rates, the proportion of interitem intervals in which rehearsal occurs decreases. Finally, longer lists always produce greater rehearsal effort.

These findings have important implications for the hypotheses of serial position effects outlined at the beginning of this paper. First, the observed cumulative rehearsal, that is, rehearsal of the list to date following each item presentation, provides more rehearsals of earlier items than of later ones. This factor is thus a possible source of primacy effects. Second, rehearsal during the delay period was not observed to terminate at random list positions; subjects appeared able to judge the delay duration and time their rehearsals accordingly. Typically, subjects would complete one or more rehearsals of the entire list and usually allowed a short pause before the test item presentation, perhaps in preparation for responding. If this finding is reliable, the hypothesis that flat serial position curves at long delays result from averaging over variations in the serial position of the last item rehearsed must be rejected.

These guidelines were used to construct five rehearsal protocols for the reaction time predictions of the two models as shown in Figure 3. The rehearsals were arranged to reflect the different strategies as closely as possible. These are, nevertheless, artificial protocols, so caution should be taken when interpreting the results. For example, the predictions

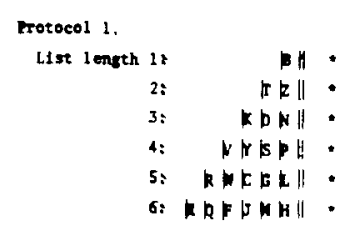

Protoeel 2

List length :

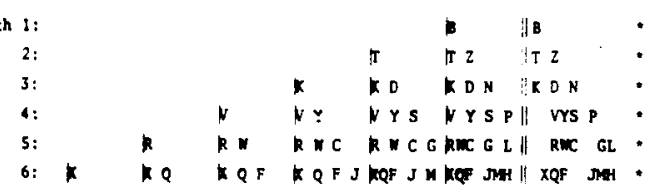

Protocol 3.

List length

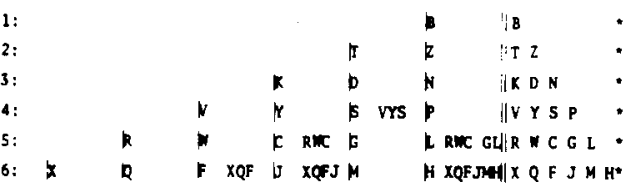

Protocol 4.

List length

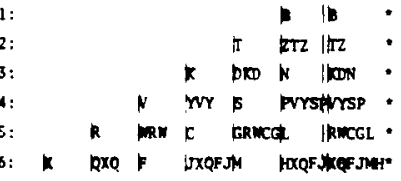

Protocol 5 .

List length

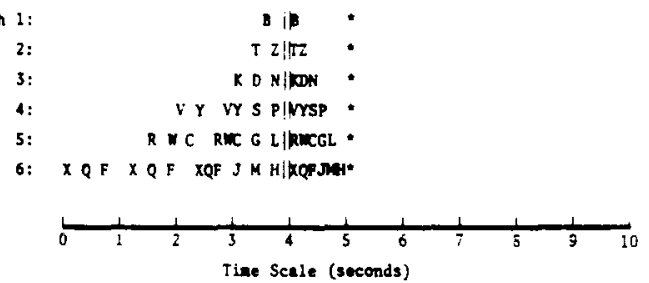

Figure 3. Rebeanal protocols used in predicting RT in the item recognilion tact. The presentation of an them is indicatod by a single vertical bar, the start of the dehy period by a doeble bar, and the teat item by an seterith. In protocel 5, all lint item are

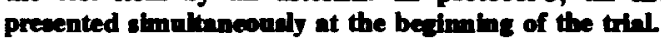

here are for only a single trial at each list length and serial position, whereas reported results are averaged over many trials and probably many rehearsal variations.

Maximum rehearsal rate was taken as 6 items/sec [Landauer (1962) reported a rate of 4.2 letters $/ \mathrm{sec}$ and Weber and Bach (1969), 6.5 letters/sec for implicit recital of the alphabet], and only multiples of $.167 \mathrm{sec}$ were used for interrehearsal spacings. In Figure 3, the presentation of an item is indicated by a single vertical bar, the start of the delay period by a double bar, and the presentation of the test by an asterisk. Protocol 1 shows a very rapid sequential presentation where it has been assumed that no rehearsal took place. Protocol 2 involves a relatively slow sequential presentation, $1.333 \mathrm{sec} /$ item, with a long delay interval of 2 sec. Cumulative list rehearsal occurs in each interitem inverval with an extra whole list rehearsal starting at the beginning of the delay period. Protocol 3 repeats the same conditions as 2 but with more relaxed rehearsal-sporadic rehearsing starts 
only after at least three items have been presented. Protocol 4 is at a faster presentation rate, $.833 \mathrm{sec} /$ item, with a short delay, $1 \mathrm{sec}$. A list rehearsal follows every second item presentation and one list rehearsal occurs during the delay period. Protocol 5 represents a simultaneous presentation in which the subject has as long as desired to study the list before pressing a ready button to initiate a $1-\mathrm{sec}$ delay period.

\section{RESULTS}

Both models achieved a linear relation between reaction time and list length, and this linearity was not affected to any great extent by variations in the free parameters of the models. These parameters were then estimated by simple trial and error to meet the following two criteria: approximately equal positive and negative slopes in protocols involving rehearsal (2 through 5-positive slope slightly greater than the negative); an average overall slope of about $40 \mathrm{msec} / \mathrm{item}$ in protocols 2 through 5 [in a survey of item recognition studies (Cavanagh, 1972b), the average slope for letter stimuli was $40.2 \mathrm{msec} / \mathrm{item}$ ]. With the free parameters thus set to generate data representative of the task under conditions of slow list presentation, both models then predicted substantially steeper positive than negative slopes in the rapid-presentation/no-rehearsal condition of protocol 1 .

The results are shown in Figure 4 for the holographic model and Figure 5 for the ETS model. The additive constants $\mathrm{a}^{+}$and $\mathrm{a}^{-}$were set at 150 and $220 \mathrm{msec}$, respectively. These constants are the same for both models in all protocols and therefore any intercept variation is a result of memory factors alone. No interpretation of intercepts will be made. however. The effects of rehearsal variations on the slope and serial-position predictions will be considered separately for the two models.

\section{Holographic Model}

As there is only one free parameter in this model. there is no guarantee that both criteria can be met simultaneously. In fact, the model is fairly successful. The value of $\alpha(.18)$ that yields positive slopes slightly greater than negative slopes in protocols 2 through 5 also yields an average slope value of $36.4 \mathrm{msec} / \mathrm{item}$ : $37.9 \mathrm{msec} /$ item for positives and $34.9 \mathrm{msec} /$ item for negatives.

In protocol 1, we find two things. First, the positive slope is about $33 \%$ greater than the negative and, second, the serial position curves do show a recency effect. However, the slope values in protocol 1 for an $\alpha$ of .18 are 20 and $15 \mathrm{msec} /$ item for positives and negatives, respectively. While these values are close to those reported by Aube and Murdock $(1974 ; 23$ and $16 \mathrm{msec} /$ item). they are about half those found by
Corballis et al. (1972) and Forrin and Morin (1969). Increasing $\alpha$ to .44 results in slopes comparable to those of the latter two studies (see Figure 4a), again with positive slope $33 \%$ greater than the negative. Further variation of $\alpha$ showed that the positive slope is always $30 \%$ to $40 \%$ steeper than the negative when there is no rehearsal.

It is not clear what experimental variations caused the difference between the slope values reported by Aube and Murdock and those found by Forrin and Morin and Corballis et al., but there are at least two possible reasons for allowing, if necessary, a higher value of $\alpha$ in protocol 1 than in protocols 2 through 5 . First, subjects may, in fact, rehearse at the very rapid presentation rate but have time only for repetition of the just-presented item. This equal amount of additional storage for each item would be reflected in a higher value of $\alpha$. Second, the rehearsal instructions may have induced the subjects to rehearse more often than would normally be the case, inflating the total amount of rehearsal assumed in protocols 2 through 5 and consequently reducing the value of $\alpha$ found in matching the predicted RT to the two criteria. However, neither of these alternatives can be accepted without further experimentation and a better understanding of the discrepancy in results among the three rapid presentation studies.

The three different rehearsal strategies in protocols 2. 3 , and 5 produced remarkably similar RT functions, as shown in Figures $4 c$ through $4 \mathrm{j}$. All have approximately the same positive and negative slopes and fairly flat serial position curves. These predictions are in accord with the findings (see Table 1) showing little difference between sequential and simultaneous presentation. The holographic model thus appears quite stable in the face of rehearsal variations that might be expected in these two presentation conditions. Furthermore, flat serial position curves are obtained even though the whole list rehearsals used in these protocols (the last item rehearsed just prior to the probe is always the last list item) provide the severest test of a recency effect.

Protocol 4 (Figures $4 \mathrm{~g}$ and $4 \mathrm{~h}$ ) represents rehearsal for a moderately rapid presentation rate and a short delay. Here, the positive and negative slopes have increased slightly in comparison with those for protocols 2 and 3 . This is in agreement with the results of Burrows and Okada (1971). In that study, a change in presentation rate from $1.5 \mathrm{sec} / \mathrm{item}$ to $.5 \mathrm{sec} /$ item resulted in a $6-\mathrm{msec} /$ item increase in both positive and negative slopes. This increase was not significant, but is in the same direction as the smaller increase of about $3 \mathrm{msec} /$ item predicted for a change from 1.333 to $.833 \mathrm{sec} /$ item by the holographic model.

With the shorter delay of this protocol, the holographic model does not, however, predict any recency effect. In addition. the recency effect found in 

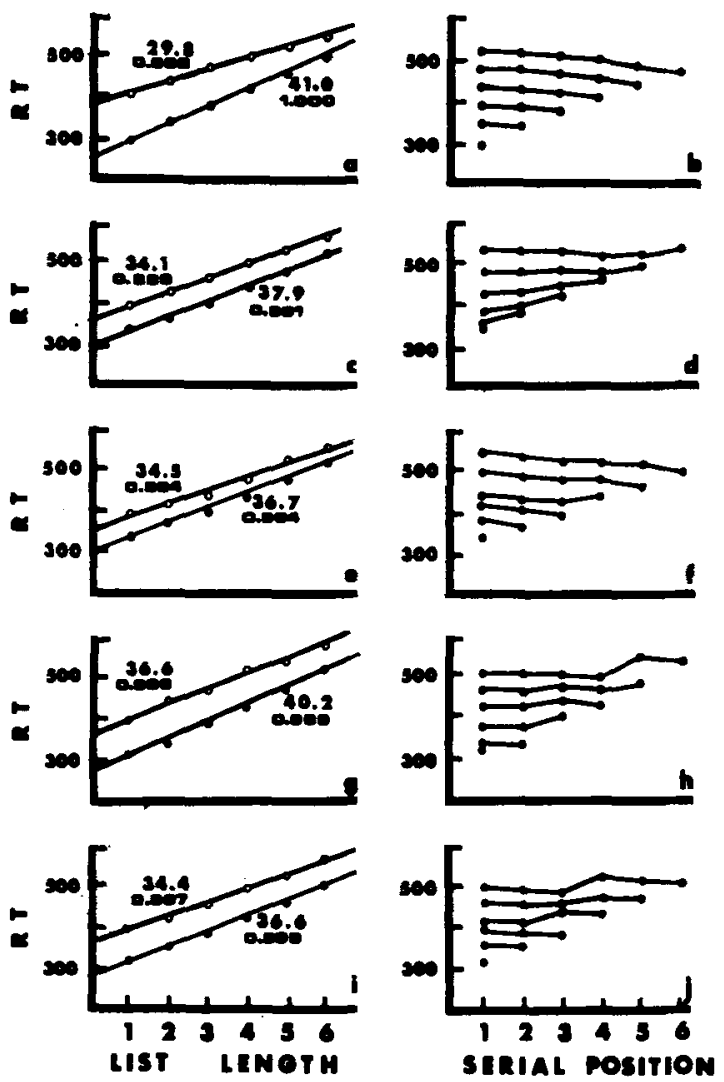

Figare 4. Reaction time predictions of the bolographic model. Pandis a, c, e, g, and I show the average podtive (filled circles) and negattve (unfilled circles) RT in millicecond as a function of tht length for protocols 1 through 5, reapectively. Also given are the slopes of the linear regreavion in millheconds/item and the $r^{2}$ values (in smaller type). Panch b, d, f, h, and $j$ show rerial poeition effects for protocols 1 through 5, respectively. The value of $a \leqslant .44$ in protocol 1 and .18 in protocols 2 to 5 .

protocol 1 (Figure $4 \mathrm{~b}$ ) is much smaller than that reported by Corballis et al. (1972) or Aubé and Murdock (1974). For example, the difference between the RT to the fifth and sixth serial positions is $18 \mathrm{msec}$ for the holographic model compared to the empirical findings of $100 \mathrm{msec}$ (Corballis et al., 1972) and $28 \mathrm{msec}$ (Aubé \& Murdock, 1974). Clearly, then, the decay of information in the holographic memory model is insufficient to account for reported recency effects.

In addition, there is no indication in protocol 1 (Figure 4b), or protocol 4 (Figure $4 \mathrm{~h}$ ) of a strong primacy effect. In protocols 2 through 5 , the flat serial position curves result from the balancing of a recency effect (evidenced in protocol 1) against a primacy effect due to extra rehearsals accorded earlier items in the list. Thus there is indirect evidence for the hypothesis, also supported by recall data (Rundus \& Atkinson, 1970), that some primacy results from extra rehearsal of earlier items. However, this certainly cannot account for the large primacy effect reported by Corballis et al. (1972; a 115-msec increase between serial position one and two, list length six), or even that found by Burrows and Okada $(1971 ; 30 \mathrm{msec}$ between positions one and two, list length four, fast presentation rate). The rehearsal hypothesis of the primacy effect therefore appears inadequate in accounting for the strong primacy at serial position 1 found in RT data. It can only be concluded, then, that the effect is due to a greater degree of learning of the first item in a list upon its initial presentation, as has been shown directly by Wickelgren (1971) and Wickelgren and Norman (1966). Since this extra learning involves only the first serial position, its effect on the average positive RT will be greater at short list lengths-especially, of course, list length one. It would thus produce a negatively accelerated positive response function, such as Briggs (Note 4) has found in many studies. Even at slow presentation rates, the extra learning would still be significant at short list lengths. At longer lengths, however, additional rehearsals would diminish the relative contribution of the initial presentation and the increased delay would further mask its effect. The fast RT to serial position one would thus be largely eliminated at slow presentation and long list length. Its presence at short list length would, nevertheless, maintain the negative acceleration of the positive RT function.

\section{ETS Model}

Of the five free parameters of this model (ignoring $\mathrm{a}^{+}$and $\mathrm{a}^{-}$), three- $\rho$, the size of the stimulus population. $y$, the response criterion position, and $t^{\prime}$. the overall decay effect of the test item and intertrial interval-have no significant effect on reaction time slopes or serial position curves. The two remaining parameters, $k_{1}$ and $k_{2}$, the relative influences of absolute trace strength and of trace strength to criterion difference on RT, were set to meet the two criteria of approximately equal positive and negative slopes and overall average slope of $40 \mathrm{msec} / \mathrm{item}$. The resulting values of $k_{1}$ and $k_{2}$ were 9.6 and 348.0, respectively. Although $k_{1}$ appears insignificantly small compared to $k_{2}$, elimination of $k_{1}\left(k_{1}=0\right)$ produces positive slopes $20 \%$ to $50 \%$ steeper than negatives in protocols 2 through 5 . Therefore, in the context of this model in the item recognition task, the hypothesis of Gescheider et al. (1969) and Pike and Ryder (1973) that latency in signal detection models is a function not only of the signal to criterion difference but also of absolute signal intensity is supported.

Now, looking at protocol 1, positive slope is much steeper than negative (Figure 5a). The conclusion is again reached that while cumulative rehearsal can lead to equal positive and negative slopes, the absence of rehearsal always produces unequal slopes. The slope magnitudes here, 46.6 and $30.3 \mathrm{msec} /$ item for positive and negative responses, respectively, are quite close to those found by Corballis et al. (1972) and Forrin and Morin (1969). The recency effect is very large (Figure 5b), comparable to that reported by 

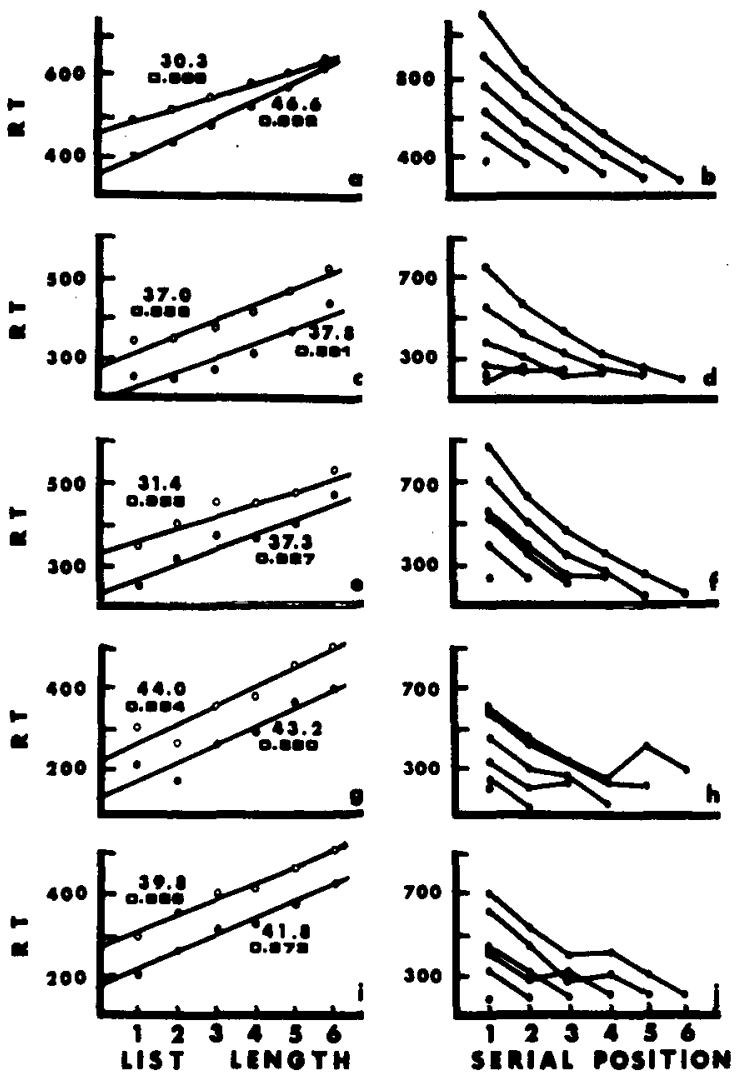

Figure 5. Reaction time predictions of the ETS model. Panels a, $c, e, g$, and $i$ show average positive (filled circles) and negative (unfilled circles) RT in milliseconds as a function of list length for protocols 1 through 5, respectively. The slopes of the linear regressions are given in milliseconds/item and $r^{2}$ values are shown in smaller type. Panels $b, d, f, h$, and $j$ show the serial position effects for protocols 1 through 5, respectively. Note the RT scale changes between the various panels. In all protocols, $\rho$, the stimulus population size is $26, \gamma$, the criterion position is .5 , i.e., midway between positive and negative averages, and $t$ ' is $1.333 \mathrm{sec}$.

Corballis et al. (1972). Furthermore, the reaction time for the last item in the list decreases with list length, as was found by Corballis et al. (1972), although there the effect is seen only for list lengths three through six. Two factors in the ETS model combine to produce this effect. First, since item trace strengths are independent, the number of items preceding the last one has no influence on its trace strength. Second. as the number of items in the list increases, the average trace strength of the positive items decreases. Since the response criterion is placed midway between the average positive strength and the average negative strength (also decreasing), the distance from the criterion to the trace strength of the last list item will increase with list length and RT will decrease. The prediction of this effect thus appears to be direct support of axiom 4 of the ETS model which allows for the deternination of a new criterion in each trial.

In protocols 2 through 5 (Figures $5 c$ to $5 \mathrm{j}$ ). the degree of linearity achieved is less than that found for the holographic model-an average $r^{2}$ of .929 vs. .992 .
Furthermore, the slopes are much more variable in response to rehearsal differences. Both protocols 2 and 4 show positively accelerated RT functions and. at places, RT decrements are predicted for increases in list length. However, such variability is not unknown experimentally, especially in individual subject data which are more likely to reveal the effects of a consistent rehearsal strategy.

Protocol 4 (Figure 5g) shows that the ETS model predicts a slight increase in slope at the faster presentation rate and short delay here as compared to protocols 2 and 3, again in agreement with Burrows and Okada (1971). Moreover, it also predicts a recency effect (Figure $5 \mathrm{~h}$ ). However, the effect is far too large, and this is the general failing of the model in all of the protocols involving rehearsal. Where the serial position curves are expected to be flat-protocols 2, 3, and 5-an overall recency effect of from 500 to $700 \mathrm{msec}$ is found at list length six. Whatever primacy effect has been introduced by the extra rehearsal of earlier items is swamped by the recency effect.

Modification of the axiom of independence-an item's trace strength is not influenced by the number of other items in memory-could reduce the recency effect. However, this axiom is not only fundamental to the model but is also instrumental in obtaining the good agreement between the magnitude of the serial position effect in protocol 1 and experimental data. The assumption of linear addition of trace strengths has been independently supported by other data (Cavanagh, Note 2); therefore, it, too, is a poor choice for modification. The most promising candidate for change is the assumption that all intervening items, both repetitions and first acquisitions, produce equal trace strength decay. One possible variant is that an item's own repetitions do not cause decay of its trace strength. However, this modification leads to an insignificant reduction in recency. The second possible variant is that first acquisitions are more effective decay-causing events than repetitions. Whereas this solution can produce flat serial position curves, it also produces an overall decrease in RT with increasing list length.

Thus, it is not possible to alter the ETS model to obtain tlat serial position curves unless the assumption of whole list rehearsal (rehearsal during the delay period always terminating with the last list item) is abandoned. For strategies involving a random terminating position, the large recency effect vanishes in averaged data. Studies of overt rehearsal in recall tasks (Corballis, 1966, 1969; Murray, 1967) have. however, shown no evidence of such strategies.

Finally, while the ETS model predicts recency effects of the correct order of magnitude in protocol 1 , the positively accelerated form of the effect (Figure 5b) is directly opposite to that found experimentally. Now the absence of the large primacy effect reported by Corballis et al. (1972) again leads to 
the assumption that the first item in a list must attain a greater trace strength upon its initial presentation than do subsequent items. This would also affect average positive RT, mostly at short list lengths, as explained previously. The positively accelerated $\mathrm{RT}$ / list-length relation for positive resonses seen in protocols 2 and 4 (Figures $5 \mathrm{c}$ and $5 \mathrm{~g}$ ) might therefore change to a linear, if not a negatively accelerated, relation. However, extra learning of the first list item would do little to alter the shape of the serial position curve for subsequent items. To change the positive acceleration of the recency effect to a negative acceleration, it would be necessary to assume occasional, partial rehearsal of the list. perhaps during the $1 / 2-$ sec delay period, that from time to time reached at least the third-to-last item in the list. Whether such rehearsal occurs is only speculation at the moment.

\section{CONCLUSIONS}

Both models supported the hypothesis that equal learning time per item at all list lengths, such as would occur in the absence of rehearsal assumed for the very fast presentation rates used by Aubé and Murdock (1974), Corballis et al. (1972), and Forrin and Morin (1969). leads to a positive slope substantially steeper than the negative. At slower presentation rates, where the average number of rehearsals per item was found to increase with list length. the positive and negative slopes in the models are approximately equal. This is a clear indication of the substantial effect rehearsal strategies may have on reaction times.

Both models also showed that the extra rehearsal of early items in the list generates only a modest degree of primacy and certainly does not account for the range of primacy effects seen experimentally. Although the cumulative rehearsal hypothesis of the primacy effect has been supported by recall data (Rundus \& Atkinson, 1970), it is not clear that it was ever a reasonable hypothesis for $\mathrm{RT}$ data. The largest primacy effects are seen under conditions of rapid presentation where rehearsal is unlikely. With slow presentation, where there is greater opportunity for extra rehearsing of early items, no primacy effects are reported. On the other hand, the hypothesis that there is a greater degree of learning, upon initial presentation, of the first item in the list (Wickelgren, 1971; Wickelgren \& Norman, 1966) is consistent with this pattern of results. In addition, the effect on average positive RT of the higher level of acquisition of the first item is greatest at short list lengths. It was seen, therefore, as a possible source of the negative acceleration of the positive RT function that is often reported.

While both models were fairly successful in predicting a wide spectrum of reaction time effects, both also suffered some shortcomings. The holographic model underestimated the slope values found in two of the three rapid presentation experiments. This may indicate either that the rehearsal protocols were unrepresentative of naturally occurring rehearsal or that the assumptions of the model were in error. The holographic model also failed to predict strong recency effects. The interactive nature of the exponential storage accounts for this-each memory record is influenced by all others in memory, tending to reduce large variations in storage levels.

The ETS model predicted large recency effects in protocols 2 through 5, where none is expected, and positively instead of negatively accelerated recency effects in protocol 1. Both of these faults may be remedied by assuming covert rehearsal strategies other than those proposed here on the basis of overt rehearsal patterns: a random variation of the last rehearsed serial position would flatten recency effects in protocols 2 to 5; occasional. partial rehearsal at the rapid presentation rate would produce a negatively accelerated recency effect in that protocol. The validity of this model thus depends on the verification of these covert rehearsal patterns.

No change in rehearsal strategy can augment the moderate recency effects found for the holographic model. It is necessary to postulate a sensory memory preceding the short-term storage simulated here, and having a much faster decay rate. The strength of the test item representation reaching the short-term storage $\left(A_{V}\right.$ in Equation 7$)$ would consequently vary strongly as a function of its presentation position, thus generating a significant recency effect.

One pattern of reaction time data remains that is at odds with any possible modification of the holographic model. Since the strength of a stored association is reduced by other associations that follow or precede it, it is impossible to produce reaction times to the last serial position that decrease with list length as seen in the data of Burrows and Okada (1971) and Corballis et al. (1972). It is likely, however, that this decrease is an artifact resulting from the uncertainty of the warning signal in these two experiments (list length varied randomly between trials). As the number of items presented approaches the maximum list length, the expectation of the warning signal, and thus its effectiveness at the short delays used, increases. When the uncertainty is removed by presenting trials in blocks of constant list length, for example (Chase, Note 5, presentation rate $.5 \mathrm{sec} /$ item, delay .25 sec, list lengths 3 and 6 ), the effect disappears.

Overall, then, the rehearsal hypothesis allowed both models to correctly predict changes in the relationship between positive and negative slopes. Serial position data, on the other hand, were not well accounted for by rehearsal variations: cumulative rehearsal generated only a small portion of the primacy effects seen in the literature; the overly large recency effects in the ETS model required that suitable partial list covert rehearsal be verified 
experimentally, while the inadequate recency effects of the holographic model suggested the participation of a sensory memory.

\section{REFERENCE NOTES}

1. Clifton, C., Jr., Gutschera, K., Brewer, E., \& Cruse, D. Recoding in a character classification task: Some inconsistent effects of recoding difficulty. Cognitive Processes Laboratory, University of Massachusetts, Amherst, 1973.

2. Cavanagh. J. P. Prediction of repetition effects in the item recognition task. Paper presented at the 6th Annual Mathematical Psychology Meetings, Montreal, August 1973.

3. Ellis. S. H. Some notes on trace strength models of item recognition. Unpublished manuscript, 1973. (Available from author, Bell Labs., Holmdel, N.J.)

4. Briggs, G. E. On the predictor variable for choice reaction time. Paper presented at the meeting of the Midwestern Psychological Association. Chicago, May 1973.

5. Chase, W. G. Research plan. Unpublished manuscript, 1974. (Available from author, Department of Psychology, Carnegie-Mellon University. Pittsburgh. Pa.)

\section{REFERENCES}

Aubé. M., \& Murdock. B. Sensory stores and high-speed scanning. Memory \& Cognition, 1974, 2, 27-33.

AXELROD, J, Noradrenaline: Fate and control of its biosynthesis. Science. 1971, 173. 598.606.

BADDELEY, A. D., \& ECOB, J. R. Reaction time and short-term memory: Implications of repetition effects for the high-speed exhaustive scan hypothesis. Quarterly Journal of Experimental Psychology. 1973, 25, 229-240.

BARRETT, T. W. Holography, information theory, and the cerebral cortex. Mathematical Biosciences, 1970, 9, 49-60.

Burrows, D., \& OKada, R. Serial position effects in high-speed memory search. Perception \& Psychophysics, 1971, 10, 305-308.

Cavanagh, J. P. Holographic processes realizable in the neural realm: Prediction of short term memory performance. (Doctoral dissertation, Carnegie-Mellon University, 1972) Dissertation Abstracts International, 1973, 33, 3280B. (University Microfilms No. 72.234, 79) (a)

Cavanagh, J. P. Relation between the immediate memory span and the memory search rate. Psychological Review, 1972. 79. $525-530$. (b)

Cavanagh. J. P., \& Chase, W. G. The equivalence of target and nontarget processing in visual search. Perception \& Psychophysics. 1971, 9. 493-495.

Clifton, C., JR.. \& Birenbaum, S. Effects of serial position and delay of probe in a memory scan task. Journal of Experimental Psychology, 1970, 86, 69-76.

Corbalis. M. C. Rehearsal and decay in immediate recall of visually and aurally presented items. Canadian Journal of Psychology, 1966, 20, 43-51.

Corballis, M. C. Serial order in recognition and recall. Journal of Experimental Psychology, 1967, 74, 99-105.

Corballis, M. C. Patterns of rehearsal iñ immediate memory. British Journal of Psychology, 1969, 60, 41-49.

Corballis, M. C.. Kirby, J., \& Miller, A. Access to elements of a memorized list. Journal of Experimental Psychology. 1972. 94. 185-190.

Eliss. S. H., \& Chase, W. G. Parallel processing in item recognition. Perception \& Psychophysics, 1971, 10, 379-384.

ForRIN. B.. \& MORIN, R. E. Recognition times for items in short- and long-term memory. Acta Psychologica. 1969. 30. 126.141.

Gescheider, G. A., Wright, J. H., Weber, B. J., Kirchier, B. M.. \& Milligan, E. A. Reaction time as a function of the intensity and probability of occurrence of vibrotactile signals. Perception \& Psychophysics, 1969, 5, 18-20.

Kirsner. K.. \& Craik, F. I. M. Naming and decision processes in short-term recognition memory. Journal of Experimental Psychology, 1971. 88. 149-157.
Landaijer, T. Rate of implicit speech. Perceptual and Motor Skills, 1962, 15, 646.

MURRAY, D. J. Overt versus covert rehearsal in short-term memory. Psychonomic Science, 1967, 7, 363-364.

Nickerson, R. S. Binary-classification reaction time: A review of some studies of human information-processing capabilities. Psychonomic Monograph Supplement, 1972, 4(Whole No. 65), 275-318.

Norman, D. A., \& Wickelgren, W. A. Strength theory of decision rules and latency in retrieval from short-term memory. Journal of Mathematical Psychology, 1969, 6, 192-208.

Pike, R., \& Ryder. P. Response latencies in the yes $/$ no detection task: An assessment of two basic models. Perception \& Psychophysics, 1973, 13, 224-232.

Rundus, D., \& Atrinson, R. C. Rehearsal processes in free recall: A procedure for direct observation. Journal of Verbal Learning and Verbal Behavior, 1970, 9, 99-105.

STERnB ERG, S. High-speed scanning in human memory. Science, $1966,153,652-654$.

STERnBerg, S. Two operations in character recognition: Some evidence from reaction-time measurements. Perception \& Psychophysics, 1967, 2, 45-53.

Swigert, C. J. Pattern identification by spatial filtering in a neuron network model. Annual Symposium Record. Systems. Man and Cybernetics IEEE, 1971, 233-240.

ThiEs, R. E. Neuromuscular depression and the apparent depletion of transmitter in mammalian muscle. Journal of Neurophysiology, 1965, 28, 427.442.

VAN HeErden, P. J. Theory of optical information storage in solids. Applied Optics, 1963, 2, 393-400.

WEBER, R., \& BACH, M. Visual and speech imagery. British Journal of Psychology, 1969, 60, 199-202.

Wescourt, K. T., \& Atkinson, R. C. Scanning for information in long- and short-term memory. Journal of Experimental Psychology, 1973, 98, 95-101.

WESTLAKE, P. R. The possibility of neural holographic processes within the brain. Kybernetik, 1970, 7, 129-153.

WICKELGREN, B. G. Habituation of spiral motoneurons. Journal of Neurophysiology, 1967, 30, 1404-1423.

WiCKelgren, W. A. Time, interference, and rate of presentation in short-term recognition memory for items. Journal of Mathematical Psychology, 1970, 7, 219-235. (a)

WiCKelgRen. W. A. Multirace strength theory. In D. A. Norman (Ed.), Models of human memory. New York: Academic Press. 1970. 67-102. (b)

WICKELGREN, W. A. Invariance of forgetting rate with number of repetitions in verbal short-term recognition memory. Psychonomic Science, 1971, 22, 363-364.

Wickelgren. W. A.. \& Berian, K. M. Dual trace theory and the consolidation of long-term memory. Journal of Mathematical Psychology, 1971, 8 404-417.

Wickelgren. W. A., \& Norman. D. A. Strength models and serial position in short-term memory. Journal of Experimental Psychology. 1966. 72. 369-381.

\section{NOTES}

1. While both models assume some form of decay, they also exhibit loss of stored information as a result of the input of new material.

2. The logarithmic detection function can also be derived from simple RT variation to stimulus intensity (Cavanagh, 1972a).

3. An alternative hypothesis here is to set the criterion between the minimum positive and maximum negative trace strengths. Results with this assumption were generally less satisfactory.

4. This would be a dangerous assumption if specific. as opposed to average, trial-to-trial sequential effects were being predicted.

(Received for publication May 5, 1975; revision received July 22 , 1975.) 